\title{
Los bajamientos vocálicos en el quechua ancashino: Un análisis fonético y fonológico
}

\author{
Vowel lowering in the Quechua of Ancash: A phonetic and phonological \\ analysis \\ WHITNEY CHAPPELL ${ }^{1}$
}

\section{RESUMEN}

Este artículo ofrece una explicación fonológica del bajamiento alofónico de las vocales del quechua hablado en el departamento de Áncash, Perú. Los datos empíricos utilizados en esta investigación proceden de la elicitación de palabras realizadas por la investigadora a un quechuahablante nativo de Áncash. Estos datos son analizados fonética y fonológicamente utilizando el programa de Praat. El análisis desarrolla el proceso de bajamiento vocálico y su condicionamiento fonológico como un caso de asimilación contextualmente determinado y concluyen en que el quechua ancashino tiene un sistema fonológico de tres vocales.

Palabras clave: quechua; bajamiento vocálico; análisis fonético y fonológico.

\begin{abstract}
This paper provides a phonological explanation of allophonic lowering of the members of the Quechua spoken in the department of Ancash, Peru. Empirical data used in this research come from the elicitation of words made by the researcher to a native Ancash Quechua speaker. These data are analyzed using phonetically and phonologically Praat program. It develops analysis process and phonological vowel lowering conditioning as a case of assimilation determined contextually, concluding that the Ancash Quechua has a phonological system of three vowels.
\end{abstract}

Keywords: quechua; vowel lowering; phonetic and phonological analysis.

\section{ICHIKLLACHAW}

Kay musyapakuyqa tsuyantsaatsimun imanawmi Anqash Piru suyuchaw qichwapa hunuluhiku wukalninkuna uranqantam. Kay musyapakuyqa yarqamushqa huk runa Anqashpa kikin qichwa shiminchaw rimankaqpiqmi, kay runapa yaskiynintaqa huk musyapakuq warmim riqitsikamun. Kay riqitsinakuyqa hunitikawan hunuluhikunawanmi analisakashqa, kaypaqmi Praat programa shutiyuq nishqawan rurakashqa. Kay analisisqa riqitsimantsik imanawmi wukalkuna uran hina imanawmi hunuluhikupiq asimilakashqantam musyatsimantsik, tsayraykurmi Anqash qichwapa hunuluhikunchawqa kimsa wukalninkunalla kapun.

Pushaq shimikuna: qichaw; wukalpa uraynin; funitikawam funulugikal analisis.

\footnotetext{
1 The Ohio State University, Estados Unidos.
} 


\section{INTRODUCCIÓN}

Aun cuando existen varias publicaciones acerca del quechua (Hintz, 1990; Parker, 1997; O’Rourke, 2009; Orr, 1975; Swisshelm, 1971; Woelck, 1973, entre otros), pocos estudios han ofrecido un análisis de los procesos fonológicos que motivan el bajamiento vocálico en el quechua. La mayoría de los diccionarios y las gramáticas describen superficialmente la distribución de los alófonos [e] y [o] en la proximidad de /q/, y 'la proximidad' no ofrece una explicación suficiente. Un análisis más riguroso de los bajamientos vocálicos nos permite comprender mejor los procesos fonológicos y articulatorios en el entorno de la consonate /q/.

Existe un desacuerdo que sigue hasta hoy entre los lingüistas y los académicos cusqueños acerca del número de vocales en el idioma, y una desconfianza mutua entre los dos grupos ha promovido aún más la discusión (Heggarty, 2006). Por un lado, los miembros de la Academia Mayor de la Lengua Quechua del Cuzco siguen sosteniendo que existen cinco vocales /i, e, a, o, u/, notando que un sistema ortográfico de cinco vocales ha sido utilizado en el quechua escrito desde el período Colonial. Solo pocos estudios han afirmado que existe una distinción fonémica entre las cinco vocales en algunos dialectos del quechua (véase Cusihuamán, 1976; Weber, 1987). Por otro lado, la mayoría de los lingüistas arguye que en el quechua hay tres vocales /i, a, u/. Las vocales medias $[\mathrm{e}, \mathrm{o}]$ son solamente alófonos de /i, u/ que ocurren en la proximidad del fonema /q/. Estos estudiosos aclaran que los quechuahablantes automáticamente pronuncian las vocales escritas /i, u/ como $[\mathrm{e}, \mathrm{o}]$ cuando se encuentren cerca de /q/ (Debate, 1987: 179; Jung y López, 1987: 588; López Flores, 1987: 9-11; Julca, 2009: 116-118, 129). En respuesta a la petición de los estudiosos cusqueños para transparencia ortográfica, los lingüistas subrayan el hecho de que la escritura y la pronunciación sean pocas veces fonéticamente idénticas, particularmente a través de dialectos. Por ejemplo, los cusqueños no pronuncian las palabras acto y apto exactamente como están escritas; en realidad pronuncian las palabras [ajto] y [afto], respectivamente (Debate, 1987: 163). La mayoría de los lingüistas proponen que las lenguas deberían demarcar los segmentos como letras diferentes cuando hacen una distinción fonémica, evitando un sistema ortográfico innecesariamente complicado, cuando se echa mano a la variación fonética.

Los que caen en el medio del debate son los lingüistas del Instituto Lingüístico de Verano (ILV), quienes reconocen que el idioma solo tiene tres fonemas /a, i, u/, y que idealmente el sistema ortográfico debería seguir el sistema fonémico. Sin embargo, también toman en cuenta las preferencias de los hablantes nativos que parecen favorecer el sistema de cinco vocales (Weber, 1987: 8), probablemente debido al contacto con el sistema ortográfico del castellano. Según Heggarty (2006), los estudiosos cusqueños están imponiendo la ortografía castellana en el quechua y enfocan sus investigaciones en los bilingües que ya están influenciados por la distinción en el castellano entre /i, $\mathrm{u} / \mathrm{y} / \mathrm{e}, \mathrm{o} /$, por un lado, y la falta de la distinción entre los dos fonemas de quechua /q/ y / k/ en el castellano, por el otro lado. Los bilingües que afirman que sí existe una diferencia significativa entre las vocales altas y medias de hecho están asimilando la /q/ de quechua a la $/ \mathrm{k} /$ de castellano y por eso no se dan cuenta del bajamiento vocálico provocado por el fonema /q/. Con la fusión de /k/ y /q/ en la mente de los bilingües, solamente se pudiera distinguir entre pares mínimos como / killa/ [kiKa] 'luna' y /qilla/ [qeKa] 'ocioso' por la primera vocal: la primera de ellas se asimila a la vocal alta del castellano /i/ y la segunda se asimila a la vocal media /e/ del castellano. Es decir, 
a pesar de que las intuiciones de los hablantes nativos sean importantes, los juicios de los bilingües de quechua y castellano parecen ser perjudicados a favor de los fonemas y la ortografía del castellano, influenciando los juicios que tienen acerca de los pares mínimos en el quechua.

El argumento a favor del sistema de tres vocales está fortalecido por la pronunciación de las palabras prestadas del castellano en el habla de hablantes monolingües del quechua quienes han tenido poco contacto con la lengua castellana. Las vocales medias de estas palabras prestadas se levantan en el habla de los monolingües, por ejemplo, dedo $>$ riru, hormiga $>$ hurminga, verde $>$ birdi (Orr, 1975). En estos casos, parece que los hablantes monolingües asimilan los sonidos castellanos a los fonemas más cercanos: las vocales altas /i/ y / u/ del quechua. A pesar de la evidencia lingüística, los académicos cusqueños hasta llegan a decir que la adopción de un sistema de tres vocales en vez de cinco equivaldría a la admisión de la inferioridad del quechua (Félix Julca, comunicación personal), que indica que los factores culturales y políticos se imponen sobre los factores lingüísticos en su análisis. Este argumento a favor del sistema de cinco vocales, entonces, se basa en criterios subjetivos, una tradición de ortografía castellana y estudios influenciados por el bilingüismo más que los hechos concretos de la lingüística de la lengua quechua.

Basada en estas observaciones y los datos que obtuvo la autora, este trabajo adopta la perspectiva de las tres vocales, ilustrando que las vocales medias demuestran una dependencia contextual con el fonema /q/. Propone la autora que este fenómeno fonológico es un caso de asimilación del rasgo [-alto] a los segmentos [+silábicos] a su alrededor.

\section{MATERIALES Y MÉTODOS}

El tipo de investigación realizado fue el descriptivo utilizando la metodología de la lingüística descriptiva. La data fue acopiada por medio de elicitación de palabras; para ello se grabó a un quechuahablante nativo del departamento de Áncash mientras leía una lista de palabras y frases aisladas. Las grabaciones se tomaron en un aula silenciosa y el hablante fue grabado con un micrófono omnidireccional incorporado en el MacBook 2010. Después se analizó los sonidos vocálicos y consonánticos usando el programa de Praat (Boersma y Weenink, 2010) para ver la primera formante (F1) de las vocales. Cuanto más baja la primera formante, más cerrada está la vocal, por ejemplo, una F1 de $315 \mathrm{~Hz}$ pertenecería a la [u] o la [i]. Cuanta más alta la primera formante, más abierta está, por ejemplo, una F1 de 450 pertenecería a la [e] o la [o].

La serie de palabras elicitadas incluyó palabras que no están en el contexto del fonema posvelar /q/ y por eso no se anticiparía ningún bajamiento vocálico y palabras que de hecho tienen una /q/ alrededor de una vocal alta, que supuestamente causaría bajamiento vocálico. Esta lista de estímulos incorporó las vocales /i/ y /u/, acento diferente en las sílabas (tónico o átono), el uso de la /k/ y la /q/, la ubicación de la /q/ entre dos vocales altas y finalmente, la posición de /q/ separada de las vocales altas por otra consonante. Se incorporaron estas diferencias para asegurarnos de que la /q/ causa el bajamiento fonológicamente, para ver si hay otras influencias en la F1 de las vocales y para ver si todos los bajamientos son iguales en su posición descendida de la lengua. Finalmente, en la medida de las formantes se midió una sección de 5 a 10 milisegundos del medio de la vocal donde las formantes aparecieran estables. Se tomó el promedio de esa corta sección para establecer la F1 de la vocal. Además del contexto 
lingüístico alrededor de la vocal, se catalogó la F1 de la vocal, la duración de la vocal y el estrés de la sílaba en sus apuntes.

\section{RESULTADOS Y DISCUSIÓN}

Según los datos obtenidos, las vocales medias nunca aparecen en las palabras quechuas que carecen del fonema /q/2. Los ejemplos enumerados en la tabla 1 no mostrarían ningún bajamiento vocálico porque no aparece el fonema /q/.

Tabla 1. Palabras del quechua ancashino sin fonema /q/ no muestran bajamiento vocálico

\begin{tabular}{|c|c|c|c|c|}
\hline urku & 'frente' & shimi & 'lengua' & 'gato' \\
\hline urkuta & 'la frente' (O.D) & shimita & 'la lengua' (O.D.) & ‘el gato' (O.D.) \\
\hline urkukuna & 'frentes' & shimikun & 'lenguas' & mishikuna 'gatos' \\
\hline
\end{tabular}

Las medidas concretas de la primera formante (F1) en las tablas 2 y 3 indican la altura mantenida de la vocal, indicada por los bajos niveles de las medidas de la F1. Es decir, estas vocales altas no se bajan si no ocurre el fonema /q/. La segunda y tercera tablas también ilustran que a pesar del contexto inicial de palabra, final de palabra o en posición interna de palabra, la vocal alta mantiene aproximadamente la misma medida de F1, fluctuando menos de $50 \mathrm{~Hz}$. Esto significa que la posición de las vocales altas dentro de la palabra y el acento tónico o átono de la sílaba que contiene esa vocal no alteran la F1 de la vocal.

Tabla 2. Medidas de los Hertzios (Hz) de la F1 de la vocal alta / u/ en un contexto sin bajamiento vocálico

\begin{tabular}{llll}
\cline { 2 - 4 } & urku'frente' & urkuta 'la frente' (O.D.) & urkukuna 'frentes' \\
\hline Primera vocal F1 & $332 \mathrm{~Hz}$ & $334 \mathrm{~Hz}$ & $325 \mathrm{~Hz}$ \\
Segunda vocal F1 & $347 \mathrm{~Hz}$ & $332 \mathrm{~Hz}$ & $300 \mathrm{~Hz}$ \\
\hline
\end{tabular}

Una prueba t de las vocales altas / u/ comparando las que están en posición tónica con las que están en posición átona no mostró una diferencia significativa: el valor p era 0.65, mucho mayor que el valor de 0.05 o menos para concluir que tenemos dos grupos distintos. Los resultados de esta prueba t indican que el acento dentro de la palabra no juega un papel en la F1 de la /u/.

Tabla 3. Medidas de los Hertzios (Hz) de la F1 de la vocal alta /i/ en un contexto sin bajamiento vocálico

\begin{tabular}{llll} 
& shimi 'lengua' & shimita 'la lengua' (O.D.) & shimikuna 'lenguas' \\
\hline Primera vocal F1 & $330 \mathrm{~Hz}$ & $295 \mathrm{~Hz}$ & $303 \mathrm{~Hz}$ \\
Segunda vocal F1 & $337 \mathrm{~Hz}$ & $335 \mathrm{~Hz}$ & $333 \mathrm{~Hz}$ \\
\hline
\end{tabular}

2 Una excepción serían las palabras prestadas de castellano en el habla de los bilingües, pero como esto es asunto del bilingüismo y no del idioma quechua, no vamos a indagar más esta excepción.

3 El uso de negrita en estas tablas indica la posición del acento de la palabra. El acento en el quechua cae en la penúltima sílaba, entonces la adición de sufijos cambia la posición del acento (véase Julca 2009: 141-142). 
Otra prueba de t comparando las vocales altas /i/ en posición tónica con las que están en una posición átona reveló la misma falta de diferencia: el valor p fue .81, aún más grande que la diferencia vista en la comparación de las /u/. Si combinamos las dos vocales altas, la /i/ y la /u/ y compararlas según su acento, tampoco vemos una diferencia significativa entre los valores de la F1: otra prueba t nos muestra un valor p de .12. Se puede concluir de estas pruebas t que los valores de F1 pueden variar ligeramente según los factores articulatorios o fonéticos, pero las vacilaciones entre las medidas de la F1 de la / u/ y la /i/ no difieren considerablemente y quedan dentro de la misma clase de vocal.

No obstante, las medidas de la F1 sí difieren considerablemente cuando el fonema /q/ ocurre directamente antes o después de una vocal alta, lo cual está mostrado en las tablas 4 y 5 .

Tabla 4. El efecto de /q/ en la vocal anterior, la /u/

\begin{tabular}{ll}
\cline { 2 - 2 } & tushuq 'bailarín' \\
\hline Primera vocal F1 & $358 \mathrm{~Hz}$ \\
Segunda vocal F1 & $498 \mathrm{~Hz}$ \\
\hline
\end{tabular}

La primera vocal alta en tushuq en la tabla 4 ocurre en una sílaba diferente de la /q/, separada por la fricativa sorda y pos-alveolar [f] y la segunda / $\mathrm{u} /$. Por consiguiente no está afectada por el fonema, que presenta medidas semejantes a la /u/, ya vistos en la tabla 2. Por el contrario, la segunda vocal ocurre directamente antes de la /q/ y presenta una F1 mucho más alta (140 Hertzios más alta), que indica menos obstrucción de la lengua en la cavidad oral. O sea, esta posición más baja de la lengua se usa en la producción de una [o] en el castellano. Un estudio de Martínez Celdrán (1995) ${ }^{4}$ explica que la F1 media de la [o] en castellano (de la voz masculina en el castellano «estándar») es $495 \mathrm{~Hz}$ y la F1 media de la [u] en castellano es $349 \mathrm{~Hz}$, y las vocales del quechua ancashino en la tabla 4 se aproximan a esos números. Parece que los académicos cusqueños han promovido el sistema de cinco vocales con tanta vehemencia precisamente porque los alófonos que preceden a la /q/ son considerablemente más bajos que las /u/ e /i/ no afectadas y se parecen mucho a las producciones de la [o] y la [e] de castellano en sus F1.

Medidas similares pero aún más exageradas aparecen en tiqti, señalando una posición apreciablemente más baja de la lengua en la primera vocal, que precede directamente a la /q/, que en la segunda, que queda separada de la /q/ por la /t/.

Tabla 5. El efecto de la /q/ en la F1 de la vocal anterior, la /i/

\begin{tabular}{ll}
\cline { 2 - 2 } & tiqti 'al estilo negro' \\
\hline Primera vocal F1 & $500 \mathrm{~Hz}$ \\
Segunda vocal F1 & $245 \mathrm{~Hz}$ \\
\hline
\end{tabular}

\footnotetext{
${ }_{4}$ Este estudio de Martínez Celdrán utilizó el habla de 5 hombres del castellano <<estándar $>>$. Entonces podría ser que los sonidos fueran diferentes de alguna manera. No obstante, en otro estudio de Morrison and Escudero (2007) los autores se encargaron de una investigación bi-dialectal y encontraron que no hay una diferencia significativa entre la F1 de los españoles y la F1 de los peruanos. Basándonos en estos datos, presumimos los valores de F1 que presenta Martínez Celdrán (1995) como un promedio apropiado para los hispanohablantes masculinos de Perú.
} 
Aquí vemos una diferencia entre las dos vocales de 255 Hertzios y, otra vez, se aproximan mucho a las F1 de la [i] y la [e] del castellano. Según Martínez Celdrán, la [i] «estándar» en la voz masculina tiene una $\mathrm{F} 1$ de $313 \mathrm{~Hz}$ y la [e] tiene un valor promedio de $457 \mathrm{~Hz}$ y vemos números semejantes en la tabla 5.

Para confirmar que sí existe una diferencia significativa entre los dos grupos -las vocales sin bajamiento y las vocales bajadas en el contexto de /q/- se utilizó adicionalmente tres pruebas más de t. Esta vez, la diferencia entre las / u/ no bajadas y las /u/ bajadas salió muy significativa: el valor $\mathrm{p}$ fue $<.001$ (4.37594E-16). La diferencia entre las /i/ no bajadas y las /i/ bajadas salió igual, con un valor $\mathrm{p}<.001$ (1.73348E-07). Finalmente, la diferencia entre todas las vocales altas no bajadas [i] y [u] y todas las vocales altas bajadas [e] y [o] mostró el mismo grado de significatividad: el valor p en esa prueba de t fue <.001 también (4.92714E-22). La conclusión importante aquí es que los dos grupos de vocales, las no bajadas y las bajadas, difieren significativamente en su altura y las bajadas solamente aparecen en contextos previsibles.

La similitud de los alófonos quechuas a las vocales /o/ y /e/ del castellano no es una justificación suficiente para postular un sistema de cinco vocales. Lo que parece ocurrir es la asimilación del rasgo [-alto], inicialmente asignado al fonema/q/ posvelar, a la vocal alta que la precede, ilustrado abajo 5 .
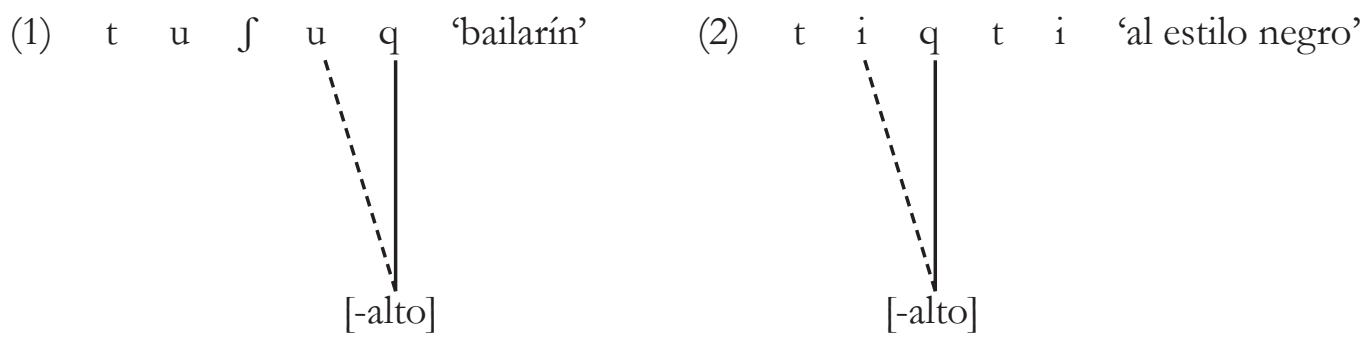

Hay que notar que la asimilación de [-alto] de la consonante /q/ a las vocales altas no es tan sencilla como la asimilación unidireccional, como la asimilación de las nasales y las laterales en el castellano. Al contrario, este bajamiento vocálico es bidireccional porque el bajamiento de las vocales altas se extiende de la derecha a la izquierda y de la izquierda a la derecha, afectando a las vocales altas en contacto directo con el fonema aunque estén antes o después de /q/. Es decir, las vocales que ocurren inmediatamente después de /q/ también experimentan el bajamiento, mostrado en las medidas de la tabla 6 .

Tabla 6. El efecto de /q/ en la vocal alta / u/ que la sigue

\begin{tabular}{ll}
\cline { 2 - 2 } & quyllur 'estrella' \\
\hline Primera vocal F1 & $447 \mathrm{~Hz}$ \\
Segunda vocal F1 & $353 \mathrm{~Hz}$ \\
\hline
\end{tabular}

Las vocales que aparecen inmediatamente antes e inmediatamente después de la consonante /q/ tienen medidas de F1 que son más de $100 \mathrm{~Hz}$, más altas que los segmen-

\footnotetext{
Este análisis fue formulado independientemente, pero apareció primero en el libro «Quechua Ancashino: una mirada actual» de Félix Julca (2009). Por consiguiente, este análisis sirve como una extensión de su explicación fonológica.
} 
tos no afectados. Cuando la /u/ baja a la [o], sus medidas son $498 \mathrm{~Hz}$ antes de /q/, como se puede ver en tushuq, y $447 \mathrm{~Hz}$ después de /q/, como se ve en quyllur, y cuando la vocal no experimenta ningún bajamiento, sus medidas son $358 \mathrm{~Hz}$ y $353 \mathrm{~Hz}$, respectivamente. Una diferencia casi igual ocurre con el bajamiento de la /i/, ilustrado abajo en la tabla $7 .{ }^{6}$

Tabla 7. El efecto de /q/ en la vocal alta /i/ que la sigue

\begin{tabular}{ll}
\cline { 2 - 2 } & qiri 'cicatriz' \\
\hline Primera vocal F1 & $417 \mathrm{~Hz}$ \\
Segunda vocal F1 & $313 \mathrm{~Hz}$ \\
\hline
\end{tabular}

Cuando la vocal / i/ en tiqti y qiri baja, la F1 aumenta desde 245 y $303 \mathrm{~Hz}$ en las vocales no afectadas hasta 500 y $410 \mathrm{~Hz}$, respectivamente, cuando preceden y siguen a /q/ directamente. Es decir, el proceso se aplica similarmente cuando la consonante /q/ causa el bajamiento de la vocal siguiente y cuando / q/ causa el bajamiento de la vocal anterior, probando la bidireccionalidad del fenómeno, ilustrado abajo en (3) y (4).

(3)

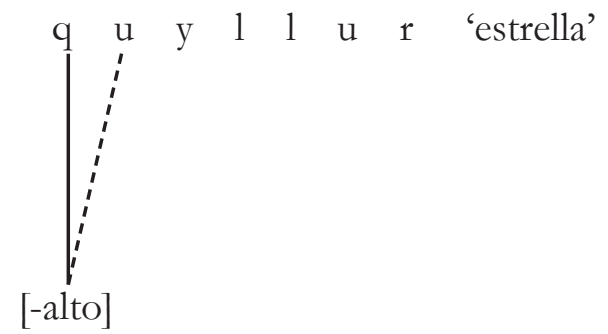

(4)

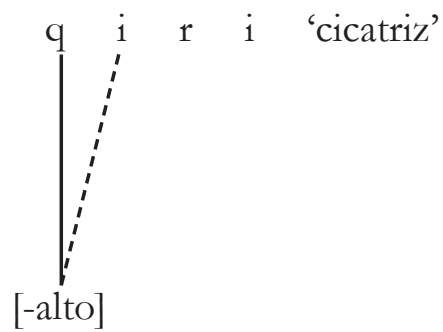

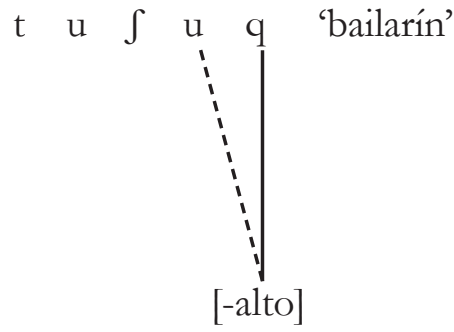

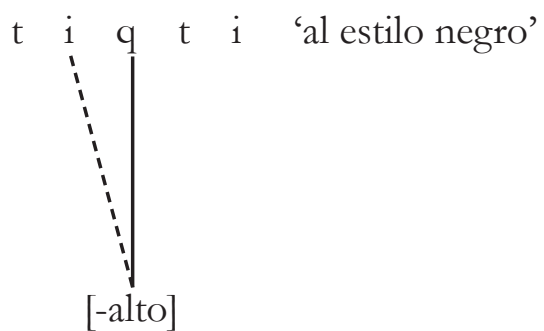

Lo más interesante de este fenómeno tiene que ver con su extensión. El proceso puede aplicarse a una vocal alta que precede a /q/ o una vocal alta que sigue a /q/; pero no siempre se aplica a las dos vocales. Si /q/ está rodeada por dos vocales, la vocal que precede a /q/ experimenta el bajamiento y la vocal posterior mantiene su F1 más baja, o sea, con una posición de la lengua más alta. Las tablas 8 y 9 demuestran la aplicación del bajamiento una vez, y la vocal que sigue a /q/ parece opaca al fenómeno.

Tabla 8. El efecto de /q/ cuando está rodeada por dos alófonos de la vocal alta /u/

\begin{tabular}{lc}
\cline { 2 - 2 } & tuqukuna 'huecos' \\
\hline Primera vocal F1 & $509 \mathrm{~Hz}$ \\
Segunda vocal F1 & $318 \mathrm{~Hz}$ \\
\hline
\end{tabular}

\footnotetext{
${ }^{6}$ Una prueba t mostró significatividad entre estos dos grupos que consisten en cuatro vocales bajadas y cuatro vocales no bajadas con un valor $\mathrm{p}$ de $<.01(0.004)$.
} 
Tabla 9. El efecto de /q/ cuando está rodeada por dos alófonos de la vocal alta /i/

\begin{tabular}{lc}
\cline { 2 - 2 } & wiqikuna 'lágrimas' \\
\hline Primera vocal F1 & $508 \mathrm{~Hz}$ \\
Segunda vocal F1 & $317 \mathrm{~Hz}^{7}$ \\
\hline
\end{tabular}

Según lo ilustrado en las tablas 8 y 9, la vocal posterior mantiene el rasgo [+alto]; no baja como la vocal que sigue a /q/ en quyllur 'estrella', anteriormente visto. Entonces, la extensión del rasgo [-alto] parece aplicarse diferentemente en este dialecto, afectando solamente la primera vocal alta si hay dos en contacto directo con la consonante /q/. Fonológicamente, podemos explicar estas diferencias en términos del bloqueo de la extensión de [-alto] a una vocal después de la /q/ si esa misma /q/ ya causó el bajamiento de una vocal anterior. Es decir, la extensión del rasgo [-alto] no es cíclica ni recurrente; ocurre una vez, representada por la línea negra, y el bloqueo de su extensión por la segunda vez se ve con la línea punteada más fina en (5) y (6).
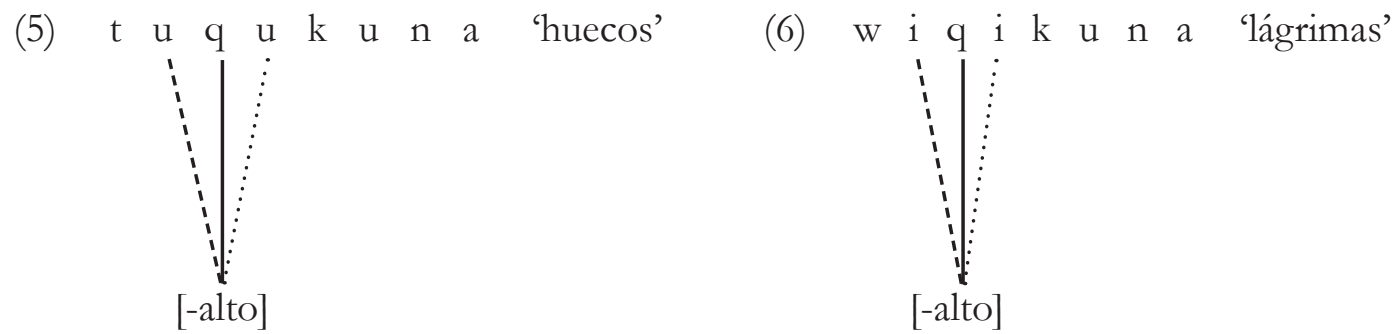

Lo mismo ocurre en palabras con dos vocales altas cuando la primera está separada de la consonante /q/ por las resonantes / $, 1, K, \mathrm{n} /$. Por ejemplo, las vocales altas en la palabra urku ya analizada cambian drásticamente cuando se reemplaza el fonema $/ \mathrm{k} /$ con el fonema /q/, ilustrado en la tabla 10. Para la conveniencia del lector, repito la tabla 2 para que se pueda comparar directamente las diferencias entre urku y urqu.

Tabla 10. Medidas de los Hertzios de la F1 de la vocal alta /u/ sin bajamiento

\begin{tabular}{cccc}
\cline { 2 - 4 } & urku 'frente' & urkuta 'la frente' (O.D.) & urkukuna 'frentes' \\
\hline Primera vocal F1 & $332 \mathrm{~Hz}$ & $334 \mathrm{~Hz}$ & $325 \mathrm{~Hz}$ \\
Segunda vocal F1 & $347 \mathrm{~Hz}$ & $332 \mathrm{~Hz}$ & $300 \mathrm{~Hz}$ \\
\hline
\end{tabular}

Tabla 11. Medidas de los Hertzios de la F1 de la vocal alta /u/ con bajamiento

$$
\text { urqu 'macho' urquta 'el macho' (O.D.) urqukuna 'machos' }
$$

\begin{tabular}{llll}
\hline Primera vocal F1 & $463 \mathrm{~Hz}$ & $403 \mathrm{~Hz}$ & $487 \mathrm{~Hz}$ \\
Segunda vocal F1 & $336 \mathrm{~Hz}$ & $328 \mathrm{~Hz}$ & $324 \mathrm{~Hz}$ \\
\hline
\end{tabular}

\footnotetext{
Una prueba t nos da un valor p significativo de $<.01(1.37055 \mathrm{E}-05)$ entre las vocales bajadas y las vocales no bajadas.
} 
La tabla 11 clarifica que el mismo proceso que ocurre con dos vocales directamente en contacto con la consonante /q/ -el bajamiento de la primera vocal y el mantenimiento de la altura de la segunda- ocurre también cuando una resonante como / r/ separa la /q/ de la vocal alta que la precede. Las resonantes son transparentes al paso del rasgo [-alto] y permiten pasarlo a las vocales altas anteriores, como se ve en (7).

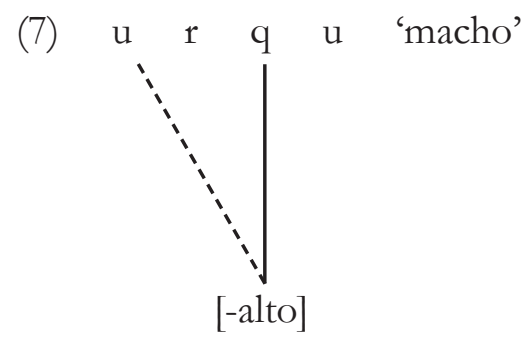

Como se observa en (7), la resonante en posición de coda es transparente a la extensión del rasgo [-alto] y permite su extensión a la vocal alta anterior. Es importante señalar que la resonante en posición de ataque no tenga la misma transparencia. De hecho, en esa posición la resonante bloquea la extensión del rasgo [-alto] y no penetra la vocal alta siguiente: el fenómeno solamente pasa de la consonante /q/ en posición de coda a la vocal alta que precede.

Hay que notar que ciertas resonantes, $/ \mathrm{r}, 1, K, \mathrm{n} /$, son transparentes al paso de [-alto] a la vocal alta anterior, pero otras resonantes, $/ \mathrm{m}, \mathrm{n} /$, no lo son. Basándonos en las pesquisas en los diccionarios y los comentarios de mi consultante del idioma, parece que los segmentos /umq/, /imq/, /unq/ e /inq/ no se permiten en la raíz de una palabra en el quechua ancashino y, por consiguiente, no pueden permitir la extensión de [-alto]. También hay que señalar que además de las resonantes $/ \mathrm{r}, 1, \kappa, \mathrm{n} /$, ciertos dialectos de Áncash-Huailas permiten variación en el bajamiento de la vocal alta en las secuencias de vocal alta + fricativa + /q/, por ejemplo, se puede pronunciar puchquy 'agriar' como [putfqui] o [potfqui]. La ocurrencia del rasgo de [-alto] en estos contextos merece más investigaciones, pero tentativamente propongo que la escala de sonoridad, vocales $>$ laterales $>$ nasales $>$ fricativas $>$ africadas $>$ oclusivas, puede explicar la transparencia del proceso. Las consonantes que son más sonoras y por consiguiente más parecidas a las vocales, i, e, las laterales y nasales, permiten el paso del rasgo [-alto], pero las que son más consonánticas, i, e, oclusivas, bloquean la extensión del rasgo. Puede ser que las fricativas y las africadas presenten una zona gris para el paso del rasgo, causando esta variabilidad que examinamos en las pronunciaciones ancashinas.

El párrafo anterior menciona el papel de la raíz de la palabra en el proceso de bajamiento, y parece que eso es un requisito del bajamiento. Como una lengua aglutinante, el quechua añade varios sufijos a la raíz de la palabra, pero la asimilación del rasgo [-alto] no puede pasar entre las fronteras de las raíces y los sufijos flexivos. Debido a esto, el fenómeno no es solamente fonológico, también es morfolexical: la /q/ y la vocal alta ha de ocurrir dentro del mismo morfema para permitir la extensión de [-alto].

Las palabras dadas abajo, por ejemplo, no experimentan bajamiento vocálico porque un sufijo que contiene /q/ se une a otro morfema -una raíz que contiene una vocal alta-. 
(8) a. hamu-rqa-ynki venir-pasado-2.PRES

'Tú viniste'

(9) а. рири-qa

ombligo-TOP

'ombligo'

(10) a. ni-nqa

decir-3.FUT

'Él/ella dirá' b. tuSu-rqa-ntsik

bailar-pasado-1PL.INCL**(i)

'Todos nosotros bailamos'

b. warmi-qa

mujer.TOP

'mujer'

b. upu-nqa

beber-3.FUT

'Él/ella tomará' c. asi-rqa-n

sonreír-pasado-3

'El/ella sonrió'

c. tampu-qa

tienda-TOP

'tienda'

c. jawi-nqa

leer-3.FUT

'Él/ella leerá'

En los ejemplos de (8), (9) y (10), queda claro que la consonante /q/ en los sufijos flexivos no afecta las vocales altas adyacentes de las raíces, que vemos con -rqa, el sufijo del pasado, -qa, el sufijo topicalizador, y-nqa, el sufijo de tercera persona del futuro. Particularmente importante es el hecho de que [-alto] no se extienda en los ejemplos de (8), (9) y (10) por razones morfoléxicas, aunque la misma secuencia de segmentos puede causar que se extienda el rasgo. Miremos, por ejemplo, sinqa 'nariz,' que ocurre en el quechua Ayacuchano, y (10a), ninqa 'él/ella dirá'. Como pares mínimos, estas palabras solamente difieren en su consonante inicial, pero sinqa constituye una sola raíz, mientras que ninqa viene del verbo niy 'decir', y está aumentado por el sufijo flexivo del futuro de tercera persona, -nqa. La vocal alta en sinqa experimenta bajamiento vocálico, pero la vocal alta en ninqa queda alta porque /q/ y la vocal alta están en morfemas diferentes, una en la raíz y la otra en el sufijo flexivo. La división entre la raíz y el sufijo prohíbe el bajamiento en ese caso.

Como era de esperar, el mismo fenómeno acontece a través de las fronteras de palabra. El mismo bloqueo creado por la distinción entre raíz y sufijo prohíbe la asimilación de [-alto] de una palabra a otra, ilustrado abajo.
(11) a. sumaq ultu
'bueno/bonito renacuajo'
b. batun qasqu 'pecho grande'
c. puwaq imiKa
'ocho chicas'

Estos ejemplos demuestran que el rasgo [-alto] que se origina en /q/ está bloqueado a través de las fronteras de morfemas. Sin embargo, si las secuencias /qu/, /unq/ y /qi/ hubieran ocurrido en el mismo morfema, sin duda habrían bajado, como es el caso en palabras como quyllur 'estrella', shunqu 'corazón' y qiri 'cicatriz'.

Aunque he mostrado que el rasgo [-alto] está bloqueado a través de morfema flexivo y fronteras de palabra, la asimilación no está bloqueada a través de morfemas derivacionales. Miremos, por ejemplo, la palabra yachachiy, 'enseñar'. Yachachiq 'maestro' se forma de ese verbo, de la raíz yachachi- y del sufijo derivacional -q, que añade el significado de 'uno que hace X'. La vocal alta precede directamente a /q/, el sufijo derivacional, y el rasgo [-alto] se extiende a la vocal. El bloqueo creado entre el raíz y los sufijos flexivos 
no se aplica a los sufijos derivacionales, y la palabra se pronuncia [yatfatfeq]. Para ir un paso más allá, el sufijo -ku se puede agregar a las ocupaciones para indicar una dedicación a largo plazo a la carrera. Entonces, yachachiq llega a ser yachachikuq, y porque el sufijo es derivacional otra vez, la asimilación hace que la / u/ baje, resultando en la pronunciación [yatfatfikoq]. Esto nos dice que la morfología juega un gran papel en el bajamiento vocálico e influencia el proceso fonológico de bajamiento vocálico.

Tenemos que recordar que no todas las reglas fonológicas se aplican iguales a las reglas de la asimilación de [-alto]; hay reglas distintas que gobiernan los procesos diferentes en el quechua. Por ejemplo, la asignación de acento no presta atención a la categorización flexiva o derivacional del sufijo como [-alto], sino se aplica indiscriminadamente en la penúltima sílaba de una palabra, demostrando la independencia de la aplicación de las reglas fonológicas. Eso es, no podemos refundir nuestros análisis de los procesos fonológicos en el quechua ancashino porque siguen reglas diferentes y tienen niveles diferentes de opacidad a la morfología.

\section{CONCLUSIÓN}

El análisis del bajamiento vocálico en el dialecto de quechua ancashino prueba que el fenómeno es mucho más complejo de lo que muchos autores habían propuesto anteriormente. Aunque la mayoría de las explicaciones fonológicas describen el bajamiento «en la proximidad de la /q/» se ofrece una explicación más detallada de los contextos donde hay asimilación del rasgo [-alto] y cuando /q/ no puede causar bajamiento vocálico. Por supuesto hay algunos académicos que no quieren admitir que las vocales medias del quechua son alófonos de la /i/ y la /u/ debido a las presiones políticas, un deseo de adaptarse al sistema vocálico del castellano e investigaciones basadas en los datos torcidos de los bilingües, pero la predictibilidad de su ocurrencia en el habla de los quechuahablantes monolingües, visible en las medidas de las primeras formantes, presenta evidencia irrefutable a favor de un sistema de tres vocales.

Este trabajo arroja cierta luz acerca de los contextos en los cuales ocurren la extensión del rasgo [-alto], pero este estudio debería ser tratado como un punto de partida para la continuación de investigaciones sobre el asunto. Primero, un análisis de más hablantes nativos del dialecto Ancashino ofrecería más evidencia apoyando el sistema de tres vocales y potencialmente revelaría otras variaciones en la extensión del rasgo [-alto]. Mi informante quechuahablante, por ejemplo, ha notado variaciones sutiles en las pronunciaciones de ciertas palabras, por ejemplo, la pronunciación de shunqu 'corazón' como

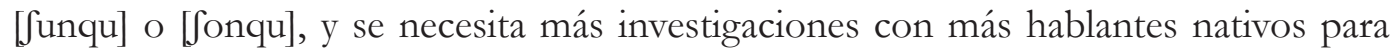
determinar si esto es un proceso fonológicamente determinado o si ocurre variación fonética en algunas palabras especificadas. Segundo, un estudio más extenso acerca de la extensión de [-alto] en varios dialectos del quechua nos otorgaría una vista del tratamiento del fenómeno y las reglas fonológicas que se aplican en dialectos aparte del Ancashino, dándonos una visión más global del quechua hablado hoy en día.

\section{REFERENCIAS BIBLIOGRÁFICAS}

Boersma, Paul and David Weenink. 2010. PRAAT: doing phonetics by computer Programa de computadora]. Versión 5.1.31 <http://www.praat.org/> [Consulta: 03-032010]. 
Cusihuaman, Antonio. 1976. Gramática Quechua: Cuz̨co-Collao (1ª Edición). Lima: Instituto de Estudios Peruanos.

Heggarty, Paul. 2006. Quechua. <http://www.quechua.org.uk/> [Consulta: 10-112010].

Hintz, Daniel. 1990. Distinctives of Corongo Quechua: Historical and synchronic perspectives. Lima: Instituto Lingüístico de Verano.

Julca, Félix. 2009. Quechua Ancashino: Una mirada actual. Lima: Fondo Editorial del Pedagógico San Marcos.

Jung, Ingrid y López, Luis. 1987. «Aportes del proyecto de educación bilingüe-Puno a la discusión en torno al alfabeto Quechua». Allpanchis 29-30: 585-92.

López, Carmen. 1987. ¿Por Qué es Mejor Escribir Con Tres Vocales en Quechua? Lima: Ministerio de Educación.

Martínez, Eugenio. 1995. En torno a las vocales del español: Análisis y reconocimiento. Estudios de Fonética Experimental 7: 195-218.

Ministerio de Educación. 1987. «Debate: ¿Por qué el uso de las tres vocales en quechua?» En Seminario Taller sobre Programas Curriculares para Educación Bilingüe: Informe Final. Lima: Ministerio de Educación.

Morrison, Geoff y Escudero, Paola. 2007. A cross-dialect comparison of Peninsular- and Peruvian-Spanish vowels. Proceedings of the International Congress of Phonetic Sciences, Saarbrucken.

O’Rourke, Erin. 2009. «Phonetics and phonology of Cuzco Quechua declarative intonation: An instrumental analysis». Journal of the International Phonetic Association 39: 291-312.

Orr, Carolyn. 1975. «Fonología del quichua del Ecuador». Estudios fonológicos de lenguas vernáculas del Ecuador. M. Catherine Peeke (ed.). Quito: Ministerio de Educación Pública. 68-86.

Parker, Steve. 1997. An OT account of laryngealization in Curco Quechua. Work Papers of the Summer Institute of Linguistics, University of North Dakota Session. Vol. 41. <http://www.und.nodak.edu/dep/linguistics/wp/1997Parker.pdf> [Consulta: 24-10-2010].

Swisshelm, Germán. 1971. Un análisis detallado de la fonología del quechua de Huaraz: Huaraz: Estudios Culturales Benedictinos.

Weber, David. 1987. Comentarios sobre la Ortografía Quechua. Manuscrito inédito.

Woelck, Wolfgang. 1973. Fonología generativa del quechua. 2da. ed. Lima: Universidad Nacional Mayor de San Marcos, Centro de Investigación de Lingüística Aplicada.

Fecha de recepción: 08 de marzo de 2015

Fecha de aceptación: 01 de junio de 2015

\section{Correspondencia}

Whitney Chappell

whitney.chappell@utsa.edu 